\title{
ENTROPY AND COLLAPSING OF COMPACT COMPLEX SURFACES
}

\author{
GABRIEL P. PATERNAIN AND JIMMY PETEAN
}

\begin{abstract}
We study the problem of existence of $\mathcal{F}$-structures (in the sense of Cheeger and Gromov, but not necessarily polarized) on compact complex surfaces. We give a complete classification of compact complex surfaces of Kähler type admitting $\mathcal{F}$-structures. In the non-Kähler case we give a complete classification modulo the gap in the classification of surfaces of class VII. In all these examples a surface admits an $\mathcal{F}$-structure if and only if it admits a $\mathcal{T}$-structure.

We then use these results to study the minimal entropy problem for compact complex surfaces: we prove that, modulo the gap in the classification of surfaces of class VII, all compact complex surfaces of Kodaira dimension $\leq 1$ have minimal entropy 0 and such a surface admits a smooth metric $g$ with $\mathrm{h}_{\mathrm{top}}(g)=0$ if and only if it is $\mathbb{C} P^{2}$, a ruled surface of genus 0 or 1 , a Hopf surface, a complex torus, a Kodaira surface, a hyperelliptic surface or a Kodaira surface modulo a finite group. The key result we use to prove this, is a new topological obstruction to the existence of metrics with vanishing topological entropy.

Finally we show that these results fit perfectly into Wall's study of geometric structures on compact complex surfaces. For instance, we show that the minimal entropy problem can be solved for a minimal compact Kähler surface $S$ of Kodaira dimension $-\infty, 0$ or 1 if and only if $S$ admits a geometric structure modelled on $\mathbb{C} P^{2}, S^{2} \times S^{2}, S^{2} \times \mathbb{E}^{2}$ or $\mathbb{E}^{4}$.
\end{abstract}

\section{INTRODUCTION}

The concept of an $\mathcal{F}$-structure was introduced by M. Gromov in [19] as a natural generalization of a torus action on a manifold. Then J. Cheeger and Gromov (19, 9, 10) related the existence of $\mathcal{F}$-structures with the possibility of collapsing a manifold with bounds on certain geometric invariants (curvature, injectivity radius, diameter, etc.). These results require the $\mathcal{F}$-structures to be of a very restrictive type, namely, polarized $\mathcal{F}$-structures. An $\mathcal{F}$-structure is a sheaf of tori acting on open subsets of a manifold (with certain compatibility on the intersection of the open subsets); polarized means (broadly speaking) that the dimension of the orbits of the actions is locally constant. In [35] the authors proved that if a closed, smooth manifold $M$ admits a general $\mathcal{F}$-structure, then one can collapse the volume of $M$ with curvature bounded from below and with bounded entropy. Namely, there exists a sequence $g_{i}$ of Riemannian metrics on $M$ such that there is a uniform upper bound on the entropy of the geodesic flow of $g_{i}$ and a uniform lower bound for the sectional curvatures of $g_{i}$,

Date: revised and improved version: June 2003.

G. P. Paternain was partially supported by CIMAT, Guanajuato, México.

J. Petean is supported by grant $37558-\mathrm{E}$ of CONACYT. 
while the volume of $\left(M, g_{i}\right)$ converges to 0 . This is our main motivation for looking at the problem of existence of $\mathcal{F}$-structures on a given manifold.

On the other hand, compact complex surfaces provide the most important family of examples of smooth closed 4-manifolds and they are fundamental in the study of differential topology in dimension 4 . Therefore it seems worthwhile to investigate which of them admit general $\mathcal{F}$-structures. There is a well-known classification of compact complex surfaces (the Enriques-Kodaira classification). The classification first divides them according to the Kodaira dimension $\kappa$, which could be $-\infty, 0,1$ or 2. We briefly recall its definition. Let $S$ be a compact complex surface and let $P_{m}(S)$ be the dimension of the space of holomorphic sections of the $m$ th tensor power of the canonical line bundle of $S$. If $P_{m}(S)=0$ for all $m \geq 1$, then $\kappa=-\infty$, otherwise, $\kappa=\lim \sup _{m \rightarrow \infty} \frac{\log P_{m}(S)}{\log m}$.

Surfaces of Kodaira dimension 0 or 1 are all diffeomorphic to elliptic surfaces. Surfaces of Kodaira dimension 2 are known as surfaces of general type since these are the generic surfaces. It is for surfaces of Kodaira dimension $-\infty$ that the classification is not complete (at least not known to be complete). Kähler surfaces of Kodaira dimension $-\infty$ are obtained by blowing up $\mathbb{C} P^{2}$ or $\mathbb{C} P^{1}$-bundles over a Riemann surface and are perfectly understood. The gap in the classification appears in the non-Kähler case. Non-Kähler surfaces of Kodaira dimension $-\infty$ are called surfaces of class VII. There are quite a few examples. First, we have the Hopf surfaces, characterized by the fact that their universal cover is $\mathbb{C}^{2}-\{0\}$. Then we have the Inoue surfaces with vanishing second Betti number and some compact elliptic surfaces. Finally we have the surfaces with global spherical shells, which have positive second Betti number. These are all the known examples and it has been conjectured that these are actually all the minimal surfaces of class VII. See Section 2 for more details. The first aim of this article is to prove the following results:

Theorem A. Let $M$ be a compact Kähler surface. Then $M$ admits an $\mathcal{F}$-structure if and only if the Kodaira dimension of $M$ is different from 2. Actually, Kähler surfaces of Kodaira number $-\infty, 0$ or 1 admit $\mathcal{T}$-structures.

Theorem B. All known examples of compact complex surfaces which are not of Kähler type admit $\mathcal{T}$-structures.

Using the result of [35] mentioned above we obtain:

Corollary. Every compact complex surface which is not of general type has minimal entropy 0 and collapses with sectional curvature bounded from below, except, perhaps, some new examples of surfaces of class VII.

We will prove these results in Sections 2 and 3. Next, we turn our attention to the problem of determining for which compact complex surfaces the minimal entropy problem can be solved. Recall that the minimal entropy of a closed manifold $M$, $\mathbf{h}(M)$, is the infimum of the topological entropy of $C^{\infty}$ Riemannian metrics on $M$ with volume one. We say that the minimal entropy problem for $M$ can be solved if there exists a $C^{\infty}$ metric $g$ with volume one for which $h_{\text {top }}(g)=\mathbf{h}(M)$. The guiding principle is that manifolds for which the minimal entropy problem can be solved 
should be topologically simple and/or support geometric structures. See [1, 35] for details and related results. In Section 5 we begin the study of the minimal entropy problem for compact complex surfaces of Kodaira dimension different from 2. From the Corollary after Theorem B it follows that $\mathbf{h}(M)=0$ for all such surfaces, modulo the gap in the classification of surfaces of class VII. Therefore the minimal entropy problem can be solved for these surfaces only if they admit a metric with vanishing topological entropy.

Finding topological obstructions to the existence of metrics with zero entropy is a subtle problem. Up to now, the known results were of two kinds. Either the fundamental group was big in the sense that it had exponential growth, or the manifold was simply connected and the exponential growth in the topology was located in the loop space homology. An important ingredient in the second case is a theorem of Gromov 15, 17] which asserts that on a closed simply connected manifold $M$, homology classes of the pointed loop space $\Omega M$ can be represented by cycles formed with paths of appropriately bounded length. In the appendix we will show that Gromov's theorem also works in the case that $M$ is compact with non-empty boundary. This will help us to deal with the case of manifolds with an infinite fundamental group of subexponential growth. Essentially nothing was known in this case. Many compact complex surfaces fall into this category (as well as many other manifolds!). More precisely, we will prove the following result. (As usual, given a space $Y$ and points $x, y \in Y, \Omega(Y, x, y)$ will denote the space of paths from $x$ to $y$.)

Theorem C. Let $M$ be a closed manifold and let $\widetilde{M}$ be its universal covering. Let $X \subset \widetilde{M}$ be a compact simply connected submanifold, possibly with boundary. Consider points $x, z \in X$ and $y \in \widetilde{M}$ and a path $\alpha$ from $z$ to $y$. Define a map $\iota: \Omega(X, x, z) \rightarrow$ $\Omega(\widetilde{M}, x, y)$ by $\iota(\tau)=\tau * \alpha$ and suppose $\iota$ induces a monomorphism in homology with coefficients in the prime field $k_{p}, p$ prime or zero. Then, for any $C^{\infty}$ Riemannian metric $g$ on $M$ there exists a positive constant $C(g)$ such that

$$
\mathrm{h}_{\mathrm{top}}(g) \geq \frac{\lambda(g)}{2}+C(g)\left(-\log R_{\Omega X, p}\right),
$$

where $R_{\Omega X, p}$ is the radius of convergence of the Poincaré series

$$
\sum_{i \geq 0} b_{i}\left(\Omega X, k_{p}\right) t^{i}
$$

The quantity $\lambda(g)$ is the volume entropy of the manifold and it is defined as the exponential growth rate of the volume of balls in $\widetilde{M}$. Manning's inequality [30] asserts that for any metric $g, \mathrm{~h}_{\text {top }}(g) \geq \lambda(g)$. It is well known that $\lambda(g)>0$ if and only if $\pi_{1}(M)$ has exponential growth. Theorem $\mathrm{C}$ does not really say much if $\lambda(g)>0$, but it is most interesting when $\lambda(g)=0$ and $R_{\Omega X, p}<1$ for some $p$.

We describe a noteworthy application of Theorem C.

Theorem D. Let $M$ be a closed manifold of dimension $n \geq 3$. Suppose that $M$ can be decomposed as $X_{1} \# X_{2}$, where the order of the fundamental group of $X_{1}$ is at least 
3. If $M$ admits a $C^{\infty}$ Riemannian metric with zero topological entropy, then $X_{2}$ is a homotopy sphere.

Theorems C and D will be proved in Section 4. Note that Theorem D is optimal. B. Totaro proved in 39] that $\mathbb{R} P^{n} \# \mathbb{R} P^{n}$ and $\mathbb{C} P^{n} \# \mathbb{R} P^{2 n}$ are diffeomorphic to biquotients and hence they have real analytic metrics with zero topological entropy.

We can now state the theorems that will give complete solutions for the minimal entropy problem for compact complex surfaces of Kodaira dimension different from 2. We phrase the solution of the minimal entropy problem in terms of 4-dimensional geometric structures. We say that $M$ admits a geometric structure if $M$ admits a locally homogeneous metric. This means that if we endow $\widetilde{M}$ with the corresponding covering metric, then $\widetilde{M}$ is a complete homogeneous space and we say that $M$ admits a geometric structure modelled on $\widetilde{M}$. In dimension 4 , the maximal geometric models have been classified by R.O. Filipkiewicz [12]. C.T.C Wall [40] studied the relationship between geometric structures and complex structures for compact complex surfaces. Wall's results will be quite useful for the proof of the two theorems below.

Theorem E. Let $S$ be a compact complex surface not of Kähler type. Modulo the gap in the classification of class VII surfaces we have: The minimal entropy of $S$ is zero and the following are equivalent:

(1) The minimal entropy problem can be solved for $S$;

(2) $S$ admits a smooth metric $g$ with $\mathrm{h}_{\text {top }}(g)=0$;

(3) $S$ admits a geometric structure modelled on $S^{3} \times \mathbb{E}^{1}$ or $\mathrm{Nil}^{3} \times \mathbb{E}^{1}$;

(4) $S$ has $\kappa=-\infty, 0$ and is diffeomorphic to one of the following: a Hopf surface, a Kodaira surface, or a Kodaira surface modulo a finite group.

Recall that a Kodaira surface is a surface $S$ with $K_{S}=\mathcal{O}_{S}$ and $b_{1}=3$. Such a surface is diffeomorphic to the product $S^{1} \times N$, where $N$ is a 3-dimensional nilmanifold.

In the Kähler case we have:

Theorem F. Let $S$ be a compact complex Kähler surface with Kodaira dimension $\kappa \leq 1$. We have: The minimal entropy of $S$ is zero and the following are equivalent:

(1) The minimal entropy problem can be solved for $S$;

(2) $S$ admits a smooth metric $g$ with $\mathrm{h}_{\text {top }}(g)=0$;

(3) $S$ admits a geometric structure modelled on $\mathbb{C} P^{2}, S^{2} \times S^{2}, S^{2} \times \mathbb{E}^{2}$ or $\mathbb{E}^{4}$ or $S$ is diffeomorphic to $\mathbb{C} P^{2} \# \overline{\mathbb{C} P^{2}}$;

(4) $S$ has $\kappa=-\infty, 0$ and is diffeomorphic to one of the following: $\mathbb{C} P^{2}$, a ruled surface of genus 0 or 1 , a complex torus or a hyperelliptic surface.

Note that $\mathbb{C} P^{2} \# \overline{\mathbb{C} P^{2}}$ is the only non-minimal surface that admits a metric of zero entropy. It is also the only surface that admits a metric with zero entropy and no geometric structure.

Acknowledgements: We thank B. Totaro for useful discussions. We also thank C. LeBrun for useful comments and a very helpful hint in the construction of retractions in Section 4. The first author thanks the CIMAT, Guanajuato, México for hospitality and support while part of this work was carried out. 


\section{2. $\mathcal{T}$-STRUCTURES AND COMPACT COMPLEX SURFACES}

We will first review the definition of a $\mathcal{T}$-structure and the results we will need about them, and then study the problem of existence of such structures on compact complex surfaces using the Enriques-Kodaira classification. We will prove here Theorems A and $\mathrm{B}$ in the introduction modulo the construction of $\mathcal{T}$-structures on surfaces of Kodaira dimension $-\infty$ that we will carry out in the next section.

Definition 2.1. A $\mathcal{T}$-structure on a smooth closed manifold $M$ is a finite open cover $\left(U_{i}\right)_{i=1, \ldots, l}$ of $M$ and a non-trivial torus action on each $U_{i}$ such that the intersections of the open subsets are invariant (through all the corresponding torus actions) and the actions commute.

The $\mathcal{T}$-structure is called polarized if the torus actions on each $U_{i}$ are locally free and in the intersections the dimension of the orbits (of the corresponding torus action) is constant. The structure is called pure if the dimension of the orbits is constant.

Remark 2.2. If two manifolds of dimension greater than 2 admit $\mathcal{T}$-structures then their connected sum also admits one (see [37, 19] for the case of polarized structures on odd dimensional manifolds and 35] for the general case). We will use this result mainly to reduce the problem of existence of a $\mathcal{T}$-structure on a compact complex surface $M$ to the case when $M$ is minimal, since non-minimal surfaces are obtained from minimal ones by taking connected sums with $\overline{\mathbb{C} P^{2}}$.

We will also use the following two results from [35]:

Theorem 2.3. If a closed smooth manifold $M$ admits a $\mathcal{T}$-structure then the minimal entropy of $M$ is 0 and $M$ collapses with curvature bounded below.

(Actually, the conclusion of the theorem still holds if one only assumes the existence of an $\mathcal{F}$-structure.)

Theorem 2.4. Every elliptic compact complex surface admits a $\mathcal{T}$-structure.

In the next section we will show that Hopf surfaces, Inoue surfaces with $b_{2}=0$ and ruled surfaces all admit $\mathcal{T}$-structures. Using this, we are in a position to prove Theorems A and B.

Minimal compact complex surfaces of Kähler type are: $\mathbb{C} P^{2}$, ruled surfaces, surfaces which are deformation equivalent to elliptic surfaces (with even first Betti number) and surfaces of general type. From Theorem 2.3 we know that if a closed manifold admits a $\mathcal{T}$-structure then it collapses with curvature bounded below, and therefore its Yamabe invariant is non-negative (see [35]). But it is known that surfaces of general type have strictly negative Yamabe invariant (see [27], for instance). Therefore they cannot admit $\mathcal{T}$-structures. All the other cases are covered by the previous comments and Theorem $\mathrm{A}$ is proved.

Minimal compact complex surfaces which are not of Kähler type are either elliptic or surfaces of class VII. We therefore only need to consider surfaces of class VII. These are the surfaces which have first Betti number equal to 1 and Kodaira dimension equal 
to $-\infty$. They were first studied by Kodaira [25. All the known examples are the following:

i) Elliptic surfaces of class VII;

ii) Hopf surfaces;

iii) Inoue surfaces with $b_{2}=0$;

iv) Surfaces with a global spherical shell.

Elliptic surfaces admit $\mathcal{T}$-structures by Theorem 2.4. We will exhibit $\mathcal{T}$-structures on Hopf surfaces and Inoue surfaces with $b_{2}=0$ in the following section. Surfaces with a global spherical shell have positive second Betti number and are all diffeomorphic to a connected sum of $S^{1} \times S^{3}$ with $b_{2}(M)$ copies of $\overline{\mathbb{C} P^{2}}$. Therefore they also admit $\mathcal{T}$-structures by Remark 2.2 .

Modulo the constructions in the following section, this finishes the proof of Theorem B.

Remark 2.5. Kodaira proved that a surface of class VII with vanishing second Betti number which contains at least a curve is either an elliptic surface or a Hopf surface. Later on Inoue [21] constructed his examples of surfaces of class VII with $b_{2}=0$ which contain no curves. He also proves that if a surface of class VII with $b_{2}=0$ and no curves admits a line bundle $F_{0}$ such that $\operatorname{dim} H^{0}\left(\Omega^{1}\left(F_{0}\right)\right)>0$ then the surface is one of his examples. Later F.A. Bogomolov [5, 6] gave a proof that such a line bundle always exists, but apparently his proof was difficult to understand. Later, J. Li, S. T. Yau and F. Zheng [28, 29] gave a much simpler proof. This result concluded the classification of surfaces of class VII with $b_{2}=0$. Surfaces with a global spherical shell have positive second Betti number and it has been conjectured [32, page 220] that every surface of class VII with positive second Betti number admits a global spherical shell. If this conjecture were true, the classification would be complete and Theorem B would cover all non-Kähler surfaces.

\section{Construction of $\mathcal{T}$-structures}

In this section we will carry out the construction of $\mathcal{T}$-structures on Hopf surfaces, Inoue surfaces with vanishing second Betti number and ruled surfaces.

3.1. Hopf surfaces. The topology of Hopf surfaces was studied by M. Kato [23. Theorem 9]. There he proves that every Hopf surface is diffeomorphic either to a product $S^{1} \times\left(S^{3} / H\right)$ or to an $S^{3} / H$-bundle over $S^{1}$ with an involution as the transition function (here $H$ is some subgroup of $G L(2, \mathbb{C})$ ). In both cases the surface has an obvious locally free (or free) $S^{1}$-action.

Therefore we can write

Lemma 3.1. Every Hopf surface admits a locally free $S^{1}$-action.

3.2. Inoue surfaces with $b_{2}=0$. There are three classes of Inoue surfaces with vanishing second Betti number; named $S_{M}, S_{N, p, q, r ; t}^{+}$, and $S_{N, p, q, r}^{-}$. They were discovered by Inoue in [21]. 
We first consider the surfaces $S_{M}$. Here $M$ denotes a $3 \times 3$ integral matrix with determinant 1 and eigenvalues $\alpha, \beta$ and $\bar{\beta}$ with $\alpha>1$ and $\beta \neq \bar{\beta}$. $M$ induces a transformation $f_{M}$ of the torus $T^{3}=\mathbb{R}^{3} / \mathbb{Z}^{3}$ (sending the class of $x \in \mathbb{R}^{3}$ to the class of $M x \in \mathbb{R}^{3}$ ) and the surface $S_{M}$ is diffeomorphic to the $T^{3}$-bundle over $S^{1}$ with $f_{M}$ as transition function. Then $S_{M}$ admits a pure polarized $\mathcal{T}$-structure of rank 3 , whose orbits are the fibers of the fibration.

Now consider the surfaces $S_{N, p, q, r ; t}^{+}$. Here $N=\left(n_{i j}\right) \in S L(2, \mathbb{Z})$ is a unimodular integral $2 \times 2$ matrix with real eigenvalues $\alpha$ and $\alpha^{-1}$, with $\alpha>1$. Then pick any integers $p, q$ and $r$ with $r \neq 0$ and a complex number $t$. These are the parameters for the surfaces $S_{N, p, q, r ; t}^{+}$. Then one picks eigenvectors $\left(a_{1}, a_{2}\right)$ and $\left(b_{1}, b_{2}\right)$ (with eigenvalues $\alpha$ and $\alpha^{-1}$, respectively) and considers the analytic automorphisms of $\mathbb{H} \times \mathbb{C}$ given by

$$
\begin{gathered}
g_{0}(w, z)=(\alpha w, z+t) \\
g_{i}(w, z)=\left(w+a_{i}, z+b_{i} w+c_{i}\right), \quad i=1,2 \\
g_{3}(w, z)=\left(w, z+\left(b_{1} a_{2}-b_{2} a_{1}\right) / r\right),
\end{gathered}
$$

where $c_{1}$ and $c_{2}$ are constants which are obtained from $N$ and its eigenvectors (see 21] for the formulas) and $\mathbb{H}=\{w \in \mathbb{C}: \operatorname{Im}(w)>0\}$. The surface $S_{N, p, q, r ; t}^{+}$is then the quotient of $\mathbb{H} \times \mathbb{C}$ by the group of analytic automorphisms generated by $g_{0}, g_{1}, g_{2}$ and $g_{3}$.

Think of $S^{1}$ as obtained from the interval $\left[0,\left(b_{1} a_{2}-b_{2} a_{1}\right) / r\right]$ by identifying its endpoints (of course, $(1 / r)\left(b_{1} a_{2}-b_{2} a_{1}\right)$ can be assumed to be positive). Then it is easy to check that the formula

$$
(\theta, w, z) \mapsto(w, \theta+z)
$$

defines a locally free $S^{1}$-action on $S_{N, p, q, r ; t}^{+}$.

Finally, let us consider the surfaces $S_{N, p, q, r}^{-}$. Here $N \in G L(2, \mathbb{Z})$ is an integral $2 \times 2$ matrix with determinant equal to -1 having real eigenvalues $\alpha>1$ and $-\alpha^{-1}$. Choose non-trivial eigenvectors $\left(a_{1}, a_{2}\right)$ and $\left(b_{1}, b_{2}\right)$ corresponding to $\alpha$ and $-\alpha^{-1}$ respectively. Fix integers $p, q$ and $r$, with $r \neq 0$, and consider the analytic automorphisms of $\mathbb{H} \times \mathbb{C}$ given by

$$
\begin{gathered}
g_{0}(w, z)=(\alpha w,-z) \\
g_{i}(w, z)=\left(w+a_{i}, z+b_{i} w+c_{i}\right), \quad i=1,2 \\
g_{3}(w, z)=\left(w, z+\left(b_{1} a_{2}-b_{2} a_{1}\right) / r\right) .
\end{gathered}
$$


Here $c_{1}$ and $c_{2}$ are constants obtained from $N, a_{1}, a_{2}, b_{1}, b_{2}, p$ and $q$ (see [21] for the formulas). Then $S_{N, p, q, r}^{-}$is the quotient of $\mathbb{H} \times \mathbb{C}$ by the group of automorphisms generated by the $g_{i}$ 's.

Consider the map $\mathbb{H} \times \mathbb{C} \rightarrow \mathbb{R}_{>0}$ given by the projection onto the second coordinate (the imaginary part of the first complex coordinate). It is easy to check that it induces a map $S_{N, p, q, r}^{-} \rightarrow S^{1}$, where $S^{1}$ is the interval $[1, \alpha]$ with its endpoints identified.

Consider the group $G_{y}$ of analytic automorphisms generated by $g_{1}, g_{2}$ and $g_{3}$. The projection is invariant under these automorphisms and the fibers of it are identified with the quotient of $\mathbb{R}^{3}$ by the action of this group (with $y=\operatorname{Im}(w)$ fixed).

There is an $S^{1}$-action on each fiber given by $\theta \cdot(\operatorname{Re}(w), z)=(\operatorname{Re}(w), \operatorname{Re}(z)+$ $\theta, \operatorname{Im}(z))$, where $S^{1}$ is now thought of as the interval $\left[0,\left(b_{1} a_{2}-b_{2} a_{1}\right) / r\right]$ with its endpoints identified.

Now $S_{N, p, q, r}^{-} \rightarrow S^{1}$ gives $S_{N, p, q, r}^{-}$a fiber bundle structure. On each fiber we have the $S^{1}$-action mentioned above. Call.$_{1}$ this action. Under the transition map this action translate to a new action which we will call.$_{2}$. If these two actions were to coincide we would have a well defined $S^{1}$-action, as for the surfaces $S_{N, p, q, r ; t}^{+}$. But in order to have a $\mathcal{T}$-structure we only need these actions to commute. Now a direct computation shows that

$$
\theta \cdot{ }_{2}(\operatorname{Re}(w), \operatorname{Re}(z), \operatorname{Im}(z))=(-\theta) \cdot{ }_{1}(\operatorname{Re}(w), \operatorname{Re}(z), \operatorname{Im}(z)) .
$$

Therefore $S_{N, p, q, r}^{-}$admits a pure polarized $\mathcal{T}$-structure of rank one.

Summarizing we can write:

Lemma 3.2. Every Inoue surface with vanishing second Betti number admits a pure polarized $\mathcal{T}$-structure of positive rank.

3.3. Ruled surfaces. Every minimal ruled surface $M$ is diffeomorphic to the projectivization of a decomposable vector bundle of rank 2 over a compact Riemann surface $S$. Moreover we can assume that one of the line bundles in the decomposition is trivial and so there exists a complex line bundle $L$ over $S$ such that $M$ is diffeomorphic to $\mathbf{P}(L \oplus \mathbb{C})$. The $S^{1}$-action on $\mathbb{C}$ then induces an $S^{1}$-action on $M$. Therefore we have:

Lemma 3.3. Every ruled surface admits a non-trivial $S^{1}$-action.

\section{Obstructions to ZERo entropy}

In this section we prove Theorems $\mathrm{C}$ and $\mathrm{D}$. We will need the following version of a result due to M. Gromov [15] for manifolds with non-empty boundary. Gromov's original proof in [15] is very short. A more detailed proof is given in [16, p. 102] but it seems unclear at certain points. There is a corrected proof in the revised an updated edition [17]. Also I. Babenko sketches a proof in [3] using Adams's cobar construction. Finally there is also a proof in [33. In all these references the manifold is assumed to have empty boundary. The proofs in the cited references work also in the case of compact manifolds with non-empty boundary with some modifications. For completeness we will sketch how to modify the proof given in [33] in the appendix. 
Theorem 4.1. Given a metric $g$ on a simply connected compact manifold $X$ (possibly with boundary), there exists a constant $C>0$ such that given any pair of points $x$ and $y$ in $X$ and any positive integer $i$, any element in $H_{i}(\Omega(X, x, y))$ can be represented by a cycle whose image lies in $\Omega^{C i}(X, x, y)$.

Let $M$ be a complete Riemannian manifold. Given $x$ and $y$ in $M$ and $T>0$ let $n_{T}(x, y)$ be the number of geodesic segments of length $\leq T$ connecting $x$ to $y$. Let $B(x, T)$ be the closed ball with center at $x$ and radius $T$. Clearly $n_{T}(x, y) \geq 1$ if and only if $y \in B(x, T)$. Moreover it is easy to see that

$$
\int_{M} n_{T}(x, y) d y=\int_{B(x, T)} n_{T}(x, y) d y=\int_{B(0, T)}\left|\operatorname{det} d_{\theta} \exp _{x}\right| d \theta .
$$

Let $(M, g)$ be a closed Riemannian manifold and let $\widetilde{M}$ be its universal covering endowed with the induced metric. Given $x \in \widetilde{M}$, let $V(x, r)$ be the volume of the ball with center $x$ and radius $r$. Set

$$
\lambda(g):=\lim _{r \rightarrow+\infty} \frac{1}{r} \log V(x, r) .
$$

Manning [30] showed that the limit exists and it is independent of $x$.

It is well known [31] that $\lambda(g)$ is positive if and only if $\pi_{1}(M)$ has exponential growth. Manning's inequality [30] asserts that for any metric $g$,

$$
\mathrm{h}_{\text {top }}(g) \geq \lambda(g) \text {. }
$$

In particular, it follows that if $\pi_{1}(M)$ has exponential growth then $\mathrm{h}_{\text {top }}(g)$ is positive for any metric $g$. This fact was first observed by E.I. Dinaburg in 11.

Given a manifold $\widetilde{M}$, a subset $X \subset \widetilde{M}$, points $x, z \in X, y \in \widetilde{M}$ and a continuous path $\alpha$ from $z$ to $y$, there is an inclusion $\iota: \Omega(X, x, z) \rightarrow \Omega(\widetilde{M}, x, y)$ given by $\iota(\tau)=$ $\tau * \alpha$. Let $I: H_{*}\left(\Omega(X, x, z), k_{p}\right) \rightarrow H_{*}\left(\Omega(\widetilde{M}, x, y), k_{p}\right)$ be the map induced in homology for some coefficient field $k_{p}$. Picking a different path from $z$ to $y$ gives a new inclusion, homotopic to the previous one. Therefore the map $I$ induced in homology is the same. Moreover, it is easy to see that the question of whether the map $I$ is injective or not is independent of the choices of $x, z, y, \alpha$. We now show:

Theorem C. Let $M$ be a closed manifold and let $\widetilde{M}$ be its universal covering. Suppose that there exists a compact simply connected submanifold $X \subset \widetilde{M}$, possibly with boundary, such that for some points $x, z \in X, y \in \widetilde{M}$ and a path from $z$ to $y$ the inclusion $\iota: \Omega(X, x, z) \rightarrow \Omega(\widetilde{M}, x, y)$ induces a monomorphism $I: H_{*}\left(\Omega(X, x, z), k_{p}\right) \rightarrow$ $H_{*}\left(\Omega(\widetilde{M}, x, y), k_{p}\right)$. Then, for any $C^{\infty}$ Riemannian metric $g$ on $M$ there exists a positive constant $C(g)$ such that

$$
\mathrm{h}_{\mathrm{top}}(g) \geq \frac{\lambda(g)}{2}+C(g)\left(-\log R_{\Omega X, p}\right),
$$

where $R_{\Omega X, p}$ is the radius of convergence of the Poincaré series

$$
\sum_{i \geq 0} b_{i}\left(\Omega X, k_{p}\right) t^{i}
$$


Proof. It is well known that for any $x \in M$ (cf. [34])

$$
\mathrm{h}_{\text {top }}(g) \geq \limsup _{T \rightarrow \infty} \frac{1}{T} \log \int_{M} n_{T}(x, y) d y .
$$

Let $p: \widetilde{M} \rightarrow M$ be the covering projection. One easily checks that given any $x \in \widetilde{M}$ we have

$$
\int_{M} n_{T}(p(x), y) d y=\int_{\widetilde{M}} n_{T}(x, y) d y=\int_{B(x, T)} n_{T}(x, y) d y .
$$

Thus for any $x \in \widetilde{M}$ we have

$$
\mathrm{h}_{\mathrm{top}}(g) \geq \limsup _{T \rightarrow \infty} \frac{1}{T} \log \int_{B(x, T)} n_{T}(x, y) d y .
$$

We will use the following lemma whose proof will be given after completing the proof of the theorem. The notation is the same as in the theorem and the comments before it. In what follows we take $x \in X$.

Lemma 4.2. There exists a constant $C(g)>0$ such that for any $T \geq C i$ and any $y \in B(x, T / 2)$, every element in the image of the map I constructed using $z=x$ and $\alpha$ a minimizing geodesic from $x$ to $y$ can be represented by a cycle in $\Omega^{T}(\widetilde{M}, x, y)$.

If $x$ and $y$ are not conjugate points, then the Morse inequalities imply:

$$
n_{T}(x, y) \geq \sum_{i \geq 0} b_{i}\left(\Omega^{T}(\widetilde{M}, x, y), k_{p}\right) .
$$

Using the lemma and the hypothesis that $I$ is a monomorphism, we obtain for any positive integer $k$ and any $y \in B(x, C k / 2)$ not conjugate to $x$,

$$
n_{C k}(x, y) \geq \sum_{i \geq 0} b_{i}\left(\Omega^{C k}(\widetilde{M}, x, y), k_{p}\right) \geq \sum_{i \leq k} b_{i}\left(\Omega(X, x, x), k_{p}\right)
$$

Let us now integrate this inequality with respect to $y \in B(x, C k / 2)$. Since $b_{i}\left(\Omega(X, x, x), k_{p}\right)$ is independent of $x$ we obtain:

$$
\int_{B(x, C k / 2)} n_{C k}(x, y) d y \geq V(x, C k / 2)\left(\sum_{i \leq k} b_{i}\left(\Omega X, k_{p}\right)\right)
$$

and hence

$$
C \limsup _{T \rightarrow \infty} \frac{1}{T} \log \int_{B(x, T)} n_{T}(x, y) d y \geq C \frac{\lambda(g)}{2}+\limsup _{k \rightarrow+\infty} \frac{1}{k} \log \sum_{i \leq k} b_{i}\left(\Omega X, k_{p}\right) .
$$

Clearly

$$
-\log R_{\Omega X, p} \leq \limsup _{k \rightarrow+\infty} \frac{1}{k} \log \sum_{i=0}^{k} b_{i}\left(\Omega X, k_{p}\right)
$$


with equality if and only if the Poincaré series of $\Omega X$ has infinitely many non-zero coefficientes. Thus

$$
\limsup _{T \rightarrow \infty} \frac{1}{T} \log \int_{B(x, T)} n_{T}(x, y) d y \geq \frac{\lambda(g)}{2}+\frac{1}{C}\left(-\log R_{\Omega X, p}\right)
$$

which together with inequality (2) completes the proof of the theorem.

Proof of Lemma 4.2. Consider $c \in H_{i}(\Omega(X, x, x))$. By Theorem 4.1 there exists a constant $C^{\prime}(g)>0$ such that for any $T \geq C^{\prime} i$ there exists a cycle $\eta$ in $\Omega^{T}(X, x, x)$ such that $\eta$ represents $c$.

Let $\alpha$ be a minimizing geodesic connecting $x$ to $y$. As before, let

$$
\iota: \Omega(X, x, x) \rightarrow \Omega(\widetilde{M}, x, y)
$$

be the map

$$
\tau \mapsto \tau * \alpha .
$$

Clearly the length $\ell(\iota(\tau))$ of $\iota(\tau)$ is given by $\ell(\tau)+d(y, x)$. Let $C=2 C^{\prime}$ and $T \geq$ $C i=2 C^{\prime} i$. If $y \in B(x, T / 2)$ we have that $\iota$ maps $\Omega^{C^{\prime} i}(X, x, x)$ into $\Omega^{C^{\prime} i+T / 2}(\widetilde{M}, x, y)$. It follows that $\iota \circ \eta$ is a cycle in $\Omega^{T}(\widetilde{M}, x, y)$. Let $[\xi]$ be the class represented by $\iota \circ \eta$ in $\Omega(\widetilde{M}, x, y)$. Then, of course, $[\xi]=I(c)$ and the lemma is proved.

We should add that I. Babenko showed in [2] that if the rational homology of the loop space of $X$ grows exponentially, then $R=R_{\Omega X, 0}$ where $R$ is the radius of convergence of:

$$
\sum_{i \geq 2} \operatorname{dim}\left(\pi_{i}(X) \otimes \mathbb{Q}\right) t^{i}
$$

Corollary 4.3. Let $M$ be a closed manifold and $\widetilde{M}$ be its universal covering. Suppose that there exists a simply connected submanifold with boundary $X$ in $\widetilde{M}$ such that the map $I: H_{*}\left(\Omega(X, x, z), k_{p}\right) \rightarrow H_{*}\left(\Omega(\widetilde{M}, x, y), k_{p}\right)$ is injective (for some $x, z \in X$, $y \in \widetilde{M}$ and a path from $z$ to $y)$ and the homology $H_{i}\left(\Omega X, k_{p}\right)$ grows exponentially for some coefficient field $k_{p}$. Then $M$ does not admit a Riemannian metric with vanishing topological entropy.

The most natural condition under which one knows that the map $I$ is a monomorphism is when there exists a retraction from $\widetilde{M}$ to $X$. Therefore we have:

Corollary 4.4. Let $M$ be a closed manifold and $\widetilde{M}$ be its universal covering. Suppose that there exists a submanifold with boundary $X$ in $\widetilde{M}$ such that $X$ is a retract of $\widetilde{M}$ and the homology $H_{i}\left(\Omega X, k_{p}\right)$ grows exponentially for some coefficient field $k_{p}$. Then $M$ does not admit a Riemannian metric with vanishing topological entropy. 
4.1. A consequence. We now want to apply the previous results to give explicit topological obstructions to the existence of metrics with vanishing topological entropy. The known results up to now deal with manifolds which have big fundamental groups (groups with exponential growth) or manifolds with very small (finite) fundamental group (if the loop space homology with coefficients in any field grows exponentially, they do not admit such a metric). We will give an obstruction which can be applied to a great variety of examples in the middle range (manifolds with infinite fundamental groups with subexponential growth).

Theorem D. Let $M$ be a closed manifold of dimension $n \geq 3$. Suppose that $M$ can be decomposed as $X_{1} \# X_{2}$, where the order of the fundamental group of $X_{1}$ is greater than 2. If $M$ admits a $C^{\infty}$ Riemannian metric with zero topological entropy, then $X_{2}$ is a homotopy sphere.

Proof. First note that the fundamental group of $M$ must have subexponential growth. It is a fact from combinatorial group theory (which follows immediately from the existence of normal forms for free products, for instance) that if $A$ and $B$ are two finitely generated groups, then the free product $A * B$ contains a free subgroup of rank two unless $A$ is trivial or $B$ is trivial, or $A$ and $B$ are both of order two. Since the fundamental group of a connected sum is the free product of the fundamental groups of the summands, we conclude that $X_{2}$ must be simply connected, since the order of the fundamental group of $X_{1}$ is at least 3 .

Let $\widetilde{X_{1}}$ be the universal covering of $X_{1}$. The universal covering $\widetilde{M}$ of $M=X_{1} \# X_{2}$ is identified with the connected sum of $\widetilde{X_{1}}$ with one copy of $X_{2}$ for each element of the fundamental group of $X_{1}$. We now want to find a submanifold of $\widetilde{M}$ to play the role of $X$ in Corollary 4.3. If the fundamental group of $X_{1}$ is finite then we will take $X=\widetilde{M}$. Assume now that the fundamental group of $X_{1}$ is infinite. Take any small $n$-dimensional disc $D$ in $\widetilde{X_{1}}$. Then $X=D \# 3 X_{2}$ appears as a submanifold of $\widetilde{M}$. Moreover, we claim that in this case the map $I$ constructed as before is a monomorphism. To prove this assume that it is not the case. Then there exists a cycle defining a non-trivial homology class in $H_{i}(\Omega(X, x, z))$, which bounds an $(i+1)$-cycle in $\Omega(\widetilde{M}, x, y)$. The image of this $(i+1)$-cycle is contained in some compact subset of $\widetilde{M}$. Performing connected sums at points away from this compact subset, we see that this $(i+1)$-cycle can also be considered as a cycle in $\Omega\left(\widetilde{M} \# 3 \overline{X_{2}}, x, y\right)$. Then we would have that the map $H_{*}(\Omega(X, x, z)) \rightarrow H_{*}\left(\Omega\left(\widetilde{M} \# 3 \overline{X_{2}}, x, y\right)\right)$ is not a monomorphism. But now we can construct a retraction $r: \Omega\left(\widetilde{M} \# 3 \overline{X_{2}}, x, y\right) \rightarrow \Omega(X, x, z)$ as follows: let $\hat{D}$ be an embedded disc in $\widetilde{X_{1}}$ containing $D$ so that $y \in \hat{D}$ and which is not contained in the $(i+1)$-cycle mentioned before. Of course, $\hat{D}-D$ is an annulus and there is a radial retraction $\rho: \hat{D} \rightarrow D$ sending the boundary of $\hat{D}$ to the center of $D$. We can also assume that $\rho$ sends $y$ to $z$ and the points where one performs the surgery with $\overline{X_{2}}$ to the points in $D$ where one performs surgery with $X_{2}$. The map $\rho$ then gives a retraction $\hat{\rho}: \hat{D} \# 3 \overline{X_{2}} \# 3 X_{2} \rightarrow D \# 3 X_{2}$ (note that the original retraction $\rho$ is orientation reversing outside $D$ ). The map $\hat{\rho}$ extends to a retraction from $\widetilde{M} \# 3 \overline{X_{2}}$ 
to $X$ by sending everything else to the center of $D$. But this implies that the map $H_{*}(\Omega(X, x, z)) \rightarrow H_{*}\left(\Omega\left(\widetilde{M} \# 3 \overline{X_{2}}, x, y\right)\right)$ is a monomorphism.

Up to now we have constructed in both cases (when the fundamental group of $\widetilde{M}$ is finite or infinite) a submanifold $X$ of $\widetilde{M}$ such that the corresponding map $I$ in the homology of the path spaces is a monomorphism. To apply Corollary 4.3 it only remains to show that if $X_{2}$ is not a homotopy sphere, in both cases, the homology of the path spaces grow exponentially for some coefficient field. In the second case $X$ is the connected sum of a disc with 3 copies of $X_{2}$. Then the exponential growth follows from the following lemma. In the first case $X$ is the connected sum of the universal covering of $X_{1}$ with $k \geq 3$ copies of $X_{2}$; in this case the exponential growth follows from the work of P. Lambrechts [26, Theorem 3].

Lemma 4.5. Let $M^{n}$ be a closed simply connected manifold which is not a homotopy sphere and let $N^{n}$ be a simply connected compact manifold with non-empty boundary. For a prime $p$ let $k_{p}$ be the field of integers modulo $p$. Let $X$ be the compact manifold with boundary obtained by taking the connected sum of $N$ with $k \geq 3$ copies of $M$. Then there exists a prime $p($ or $p=0)$ such that the Betti numbers of the free loop space $\Lambda(X)$ with coefficients in $k_{p}$ grow exponentially. Therefore the Betti numbers of $\Omega(X)$ also grow exponentially.

Proof. Let us first consider the free loop space $\Lambda(X)$. Our proof will be based on the work of Lambrechts in [26] and we will will make frequent use of this reference.

Given a differential graded algebra (DGA) $(A, d)$ we will consider the Hochschild homology, $H H_{*}(A, d)$, of $(A, d)$. We will only be interested in the case when the DGA is positively or negatively graded and connected $\left(A_{0}=R\right.$, where $R$ is the ground field). In this case the Hochschild homology is also positively or negatively graded. If $K$ is a simply connected $C W$-complex of finite type and $C^{*}\left(K, k_{p}\right)$ denotes the singular cochain complex of $K$ with coefficients in $k_{p}$ then

$$
H^{*}\left(\Lambda K, k_{p}\right) \equiv H H_{*}\left(C^{*}\left(K, k_{p}\right)\right)
$$

(see [22] or [20]). A quasi-isomorphism (or quism) between two DGAs is a DGAmorphism which induces an isomorphism in homology. Two DGAs are said to be weakly equivalent if there is a chain of quasi-isomorphisms connecting them. Since a quasi-isomorphism induces an isomorphism between the corresponding Hochschild homologies, two weakly equivalent DGAs have isomorphic Hochschild homologies. In summary, to prove our lemma it is enough to obtain a DGA $(A, d)$ which is weakly equivalent to $C^{*}\left(X, k_{p}\right)$ and such that $H H_{*}(A, d)$ grows exponentially (c.f. [26, Proposition 8]).

Since $M^{n}$ is not a homotopy sphere there exists a number $i_{0}, 1<i_{0}<n$, such that $\pi_{i_{0}}(M)$ is the first non-trivial homotopy group of $M$. Then $\pi_{i_{0}}(M)=H_{i_{0}}(M, \mathbb{Z})$. Actually $i_{0} \leq n / 2$ and so $i_{0}<n-1$. If $Y$ is the connected sum of $k$ copies of $M$ then $H_{i_{0}}(Y, \mathbb{Z})=k H_{i_{0}}(M, \mathbb{Z})$ (with $k \geq 3$ ). Therefore there exists a prime $p$ such that $H_{i_{0}}\left(Y, k_{p}\right)$ is a $k_{p}$-vector space of dimension $\geq k$. From now on we fix the integer $i_{0}$ and the prime $p$. 
Pick any non-zero element in $H_{i_{0}}\left(M, k_{p}\right)$. This element gives in a canonical way three linearly independent elements in $H_{i_{0}}\left(Y, k_{p}\right)$ for which the cup product between any pair of them is 0 . Let $(A, d)$ be a $\left(i_{0}-1\right)$-connected DGA weakly equivalent to the singular cochain complex of $Y$. We can moreover assume that $d$ is decomposable and that $A^{m}=0$ for all $m>n$. We can also construct $(A, d)$ in such a way that it has three homogeneous cycles $x, x^{\prime}, y$ of degree $i_{0}$ (which correspond to the cohomology classes mentioned above) such that the product of any pair of them is 0. See for instance the argument in [26, Proposition 17]. By Poincaré duality there exists in any DGA weakly equivalent to the cochain complex of $Y$ with $k_{p}$ coefficients an element of degree $n$ which is a representative of the fundamental class of $Y$ which can be expressed as a product of homogeneous elements of positive degree. Let $D$ be a small open ball embedded in $Y$. It follows that one can construct as in [26, Proposition 18] a DGA $(\hat{A}, \hat{d})$ weakly equivalent to the singular cochain complex of $Y-D$ such that :

1) $\hat{A}=A \oplus k_{p} \mathbf{x}$ as vector spaces, where $\operatorname{deg}(\mathbf{x})=n-1$.

2) the differential and product of $\hat{A}$ extend those of $A$.

3) $\mathbf{x} \cdot \hat{A}^{+}=0$.

4) $\hat{d} \mathbf{x} \in A^{n}$ is decomposable and represents the fundamental class of $Y$.

Now we will find a suitable DGA weakly isomorphic to $C^{*}\left(N-D, k_{p}\right)$, where $D$ is some small open ball embedded in $N$. Pick a 1-connected DGA $(B, d)$ weakly isomorphic to $C^{*}\left(N, k_{p}\right)$ such that $B^{m}=0$ for all $m>n$. Then we consider the DGA $(\hat{B}, \hat{d})$ defined as follows: as graded vector spaces $\hat{B}=B \oplus k_{p} \mathbf{z}$ where $\mathbf{z}$ is a new element of degree $n-1$. The product is defined by extending the product of $B$ and declaring $\mathbf{z . z}=0$ and $\mathbf{z} \cdot B^{+}=0$. It is easy to check that these choices give a well define graded algebra. To define the differential we set $\hat{d}=d$ on $B$ and $\hat{d}(\mathbf{z})=0$. The whole idea is that $\mathbf{z}$ will represent the new $(n-1)$-cohomology class represented by $\partial D$.

Now we have to prove that $(\hat{B}, \hat{d})$ is weakly isomorphic to $C^{*}\left(N-D, k_{p}\right)$.

Let $\varphi:(T V, d) \rightarrow C^{*}\left(N, k_{p}\right)$ be a minimal $T V$-model for $C^{*}\left(N, k_{p}\right)$. Let $\hat{V}=$ $V \oplus k_{p} \omega \oplus W$ be a graded vector space where $\omega$ has degree $n-1$ and the elements of $W$ are combinations of homogeneous elements of degree $\geq n$. On $T \hat{V}$ we define a differential $\hat{d}$ by $\hat{d}=d$ on $T V, \hat{d}(\omega)=0$ and $W$ and $\left.\hat{d}\right|_{W}$ are defined in a minimal way to kill all cohomology in dimensions higher than $n$. Then we extend the map $\varphi$ to a map

$$
\hat{\varphi}: T \hat{V} \rightarrow C^{*}\left(N-D, k_{p}\right)
$$

by setting $\hat{\varphi}=i^{*} \varphi$ on $T V$, where $i$ is the inclusion of $N-D$ in $N$ and $\varphi(\omega)$ is a representative of the new $(n-1)$-cohomology class represented by $\partial D$. It is easy to check that $\hat{\varphi}$ is uniquely determined by this data and it is a quasi-isomorphism.

Since $(T V, d)$ is weakly isomorphic to $(B, d)$ there exists a quasi-isomorphism $\psi$ : $(T V, d) \rightarrow(B, d)$. Now define 


$$
\hat{\psi}:(T \hat{V}, \hat{d}) \rightarrow(\hat{B}, \hat{d})
$$

by $\hat{\psi}=\psi$ on $T V, \hat{\psi}=0$ on $W$ and $\hat{\psi}(\omega)=\mathbf{z}$. It is easy to check that $\hat{\psi}$ is a quasiisomorphism. Therefore $(\hat{B}, \hat{d})$ is weakly-equivalent to $C^{*}\left(N-D, k_{p}\right)$. Moreover, $\hat{d}$ is decomposable.

Summarizing, up to now we have constructed a DGA $(\hat{A}, \hat{d})$ weakly equivalent to $C^{*}\left(Y-D, k_{p}\right)$ with $\hat{d}$ decomposable and a DGA $(\hat{B}, \hat{d})$ weakly equivalent to $C^{*}(N-$ $\left.D, k_{p}\right)$ with $\hat{d}$ decomposable. $X$ is obtained by joining $Y-D$ and $N-D$ through $\partial D$. A DGA $(F, f)$ weakly equivalent to $C^{*}\left(X, k_{p}\right)$ can then be obtained from $(\hat{A}, \hat{d})$ and $(\hat{B}, \hat{d})$ as in [26, Proposition 17]:

$$
F=k_{p} \oplus A^{+} \oplus B^{+} \oplus k_{p} \mathbf{w}
$$

where $f=\hat{d}$ on $A^{+}$and on $B^{+}$and $f(\mathbf{w})$ is the decomposable element in $A^{n}$ representing the fundamental class of $Y$. Therefore $f$ is decomposable and the elements $x, x^{\prime}, y \in A$ satisfy all the hypotheses of [26, Proposition 9]. Therefore the Hochschild homology of $(F, f)$ grows exponentially and so does $H^{*}\left(\Lambda(X), k_{p}\right)$, from the discussion at the beginning of the proof. Finally exponential growth of the homology of $\Lambda(X)$ implies the exponential growth of the homology of $\Omega(X)$ for any finite $C W$-complex by an easy argument with the spectral sequence of the fibration

$$
\Omega(X) \mapsto \Lambda(X) \rightarrow X
$$

\section{Geometric structures and entropy}

Let us begin by recalling the definition of geometric structures in general. A geometry is a complete simply connected Riemannian manifold $X$ such that the group of isometries acts transitively on $X$ and contains a discrete subgroup with compact quotient. We then say that a closed manifold $M$ admits a geometric structure modelled on $X$ if there is a Riemannian metric on $M$ such that the Riemannian universal covering of $M$ is $X$. Maximal geometric structures in dimension 4 have been classified by Filipkiewicz in [12. Wall studies the relationship between geometric structures and complex structures on 4-manifolds in [40. Given a compact complex surface we are only interested on whether the underlying smooth manifold admits a geometric structure, but not on the compatibility of the geometric structure with the complex structure.

In this section we will describe which maximal geometric structures admit models with metrics with zero entropy. We refer to [40] for a description and details of the 4-dimensional geometries.

Proposition 5.1. If $M$ admits a geometric structure modelled on one of $S^{4}, \mathbb{C} P^{2}$, $S^{2} \times S^{2}, S^{2} \times \mathbb{E}^{2}, \mathbb{E}^{4}, S^{3} \times \mathbb{E}^{1}, \mathrm{Nil}^{3} \times \mathbb{E}^{1}$ or $\mathrm{Nil}^{4}$, then $M$ admits a smooth metric $g$ with $\mathrm{h}_{\text {top }}(g)=0$. 
Proof. (1) $S^{4}, \mathbb{C} P^{2}, S^{2} \times S^{2}, S^{2} \times \mathbb{E}^{2}, \mathbb{E}^{4}$ and $S^{3} \times \mathbb{E}^{1}$ : All the Jacobi fields in these geometries grow at most linearly (in the case of $S^{4}$ and $\mathbb{C} P^{2}$ they are actually bounded), and hence all the Liapunov exponents of every geodesic in $M$ are zero. It follows from Ruelle's inequality 36] that all the measure entropies are zero. Hence, by the variational principle, the topological entropy of the geodesic flow of $M$ must be zero.

(2) $\mathrm{Nil}^{3} \times \mathbb{E}^{1}$ and $\mathrm{Nil}^{4}$ : The geometry $\mathrm{Nil}^{3}$ can be described as $\mathbb{R}^{3}$ with the metric

$$
d s^{2}=d x^{2}+d y^{2}+(d z-x d y)^{2} .
$$

Here, not all the Jacobi fields grow linearly, but they certainly grow polynomially. Again this implies that all the Liapunov exponents of every geodesic in $M$ are zero and hence the topological entropy of the geodesic flow of $M$ must be zero. For the case of $\mathrm{Nil}^{4}$ we use a result of L. Butler [7, Theorem 1.3] which asserts that for any lattice $\Gamma$ in $\mathrm{Nil}^{4}$ and any left invariant metric, the topological entropy of the geodesic flow of $\mathrm{Nil}^{4} / \Gamma$ is zero. In fact, Butler's result applies to any nilpotent Lie group that admits a normal abelian subgroup of codimension one. For general nilpotent groups, the result is just not true; examples have been given by Butler himself in 8 .

We now show that if $M$ admits a geometric structure modelled on one of the remaining geometries, namely $S^{2} \times \mathbb{H}^{2}, \mathbb{E}^{2} \times \mathbb{H}^{2}, \mathbb{H}^{3} \times \mathbb{E}^{1}, \widetilde{\mathrm{SL}}_{2} \times \mathbb{E}^{1}, \mathrm{Sol}_{0}^{4}, \mathrm{Sol}_{m, n}^{4}$ or $\mathrm{Sol}_{1}^{4}$, then $M$ cannot admit a metric of zero topological entropy. To do this, we use the next lemma, together with the fact described in Section 4, that if $\pi_{1}(M)$ grows exponentially, then $\mathrm{h}_{\text {top }}(g)>0$ for any smooth metric $g$ on $M$.

Lemma 5.2. Let $M$ be a closed orientable 4-manifold, and suppose that $M$ admits a geometric structure modelled on one of $S^{2} \times \mathbb{H}^{2}, \mathbb{E}^{2} \times \mathbb{H}^{2}, \mathbb{H}^{3} \times \mathbb{E}^{1}, \widetilde{\mathrm{SL}_{2}} \times \mathbb{E}^{1}$, Sol ${ }_{0}^{4}$, $\mathrm{Sol}_{m, n}^{4}$ or $\mathrm{Sol}_{1}^{4}$. Then $\pi_{1}(M)$ grows exponentially.

Certainly the same is true for the geometries $\mathbb{H}^{2} \times \mathbb{H}^{2}, \mathbb{H}^{4}$ and $\mathbb{H}^{2}(\mathbb{C})$, but here one has a better result: the simplicial volume of any $M$ modelled on one of these geometries will be non-zero [19].

Proof. Let $(M, g)$ be a closed Riemannian manifold. Recall that $\pi_{1}(M)$ grows exponentially if and only if $\lambda(g)>0$.

If a closed manifold $M$ admits a geometric structure modelled on $(X, G)$, then $M$ is equipped with a locally homogeneous metric $g$. Let us consider the geometries $S^{2} \times \mathbb{H}^{2}, \mathbb{E}^{2} \times \mathbb{H}^{2}, \mathbb{H}^{3} \times \mathbb{E}^{1}$. All of them have a hyperbolic space as a factor and using this, it is pretty straightforward to check that $\lambda(g)>0$ for all of them.

The geometry $\widetilde{\mathrm{SL}}_{2}$ admits the unit sphere bundle of a closed surface of genus $\geq 2$ as a compact quotient. Since the fundamental group of the latter has exponential growth, it follows that $\lambda(g)>0$ for this geometry. Thus $\lambda(g)>0$ as well for the geometry $\widetilde{\mathrm{SL}_{2}} \times \mathbb{E}^{1}$.

To deal with the solvable geometries, it suffices to exhibit for each one of them a cocompact lattice $\Gamma$ such that $\Gamma$ has exponential growth. 
Let $A \in S L(3, \mathbb{Z})$ and let $A_{t} \in G L(3, \mathbb{R})$ be a 1 -parameter subgroup with $A_{1}=A$. Let $G$ be the semidirect product $\mathbb{R}^{3} \ltimes_{A_{t}} \mathbb{R}$. Let $\Gamma_{A} \subset G$ be the cocompact lattice given by $\mathbb{Z}^{3} \ltimes_{A} \mathbb{Z}$ (the ascending $H N N$ extension of $\mathbb{Z}^{3}$ by $A$ ).

If we choose $A$ such that it has characteristic polynomial $-\lambda^{3}+m \lambda^{2}-n \lambda+1$, then $G$ will be isomorphic to $\mathrm{Sol}_{m, n}^{4}$. We may choose for example:

$$
\left(\begin{array}{ccc}
1 & 1 & 0 \\
m-n & m-2 & 1 \\
m-n & m-3 & 1
\end{array}\right)
$$

We claim that $\Gamma_{A}$ has exponential growth. To see this it suffices to use a result of J. Wolf [41], which asserts that a solvable (polycylic) group has exponential growth unless it is virtually nilpotent. Wolf also gives a criterion to check whether this holds [41, Proposition 4.4]. It amounts to whether $A$ has an eigenvalue with absolute value different from one, which is certainly the case for our choice of $A$.

To deal with $\mathrm{Sol}_{0}^{4}$ note that the lattice $\Gamma_{A}$ exists inside $\operatorname{Sol}_{0}^{4}$ if $A$ has eigenvalues $\alpha>1, \beta$ and $\bar{\beta}$ with $\beta \neq \bar{\beta}$. In fact with such a choice of $A, \Gamma_{A}$ is exactly the fundamental group of the Inoue surface $S_{A}$. As before $\Gamma_{A}$ has exponential growth by Wolf's criterion.

Finally, in the case of $\mathrm{Sol}_{1}^{4}$, it suffices to consider the cocompact lattice given by the ascending HNN extension of the Heisenberg lattice $H_{Z}$ by an automorphism given by a hyperbolic matrix in $S L(2, \mathbb{Z})$. Again, by Wolf's criterion, the lattice has exponential growth.

\section{Elliptic surfaces}

Our references for the background material in this section about elliptic surfaces are [14, 40] and references therein.

$S$ is called an elliptic surface if there is a holomorphic map $\pi: S \rightarrow C$ whose general fibre is an elliptic curve. We will (as usual) assume that $S$ is relatively minimal, i.e., no fibre contains an exceptional curve of the first kind. In fact, except when $S$ is rational this is equivalent to minimality in the usual sense.

A classification of the possible fibres of $\pi$ was given by Kodaira [24] : the cases are labeled $I_{k}(k \geq 0)$, II, III, IV, I* $(k \geq 0)$, II* $\mathrm{III}^{*}, \mathrm{IV}^{*}$. Case $I_{0}$ means that the fibre is a smooth elliptic curve and all other types are called singular. Also important to us is the notion of multiplicity. Given $p \in C, \pi^{-1}(p)$ is a multiple fibre if there exists an integer $m>1$ such that as a divisor $\pi^{*}(p)=m D$. The largest such $m$ is called the multiplicity of the fibre. Multiple fibres can only be of type $I_{k}$ for some $k$.

We will often regard $C$ as an orbifold with a $2 \pi / m_{i}$ cone point at each point $x_{i}$ corresponding to a multiple fibre of multiplicity $m_{i}$. Then the orbifold Euler characteristic of $C$ is:

$$
\chi^{\mathrm{orb}}(C)=\chi(C)-\sum_{i}\left(1-m_{i}^{-1}\right)
$$


The structure of $S$ at a smooth multiple fibre is homeomorphic to the product of a circle with a multiple fibre in a Seifert fibration of a 3-manifold.

Let $\left\{t_{1}, \ldots, t_{n}\right\}$ be the set of multiple points of $C$ and suppose each $t_{i}$ has multiplicity $m_{i}$. Given a base point $t \in C$, define the orbifold fundamental group $\pi_{1}^{\text {orb }}(C, t)$ as follows. Let $C_{0}=C-\left\{t_{1}, \ldots, t_{n}\right\}$. The group $\pi_{1}\left(C_{0}, t\right)$ contains the image of the free group $F$ on letters $\gamma_{i}, 1 \leq i \leq n$, corresponding to loops $\gamma_{i}$ in $C_{0}$ enclosing $t_{i}$ which are null-homotopic in $C$. Now set $\pi_{1}^{\text {orb }}(C, t)$ to be the quotient of $\pi_{1}\left(C_{0}, t\right)$ by the smallest normal subgroup which contains $\gamma_{i}^{m_{i}}, 1 \leq i \leq n$.

We say that an orbifold is good if it has an orbifold covering whose total space is an orbifold with no multiple points. Otherwise, an orbifold is bad. The bad 2-dimensional orbifolds are the 2-sphere with either one multiple point or with two multiple points with unequal multiplicities.

Of central importance to us is the fact that $\chi(S)$, the Euler number of $S$, is always $\geq 0$ and it vanishes if and only if there are no singular fibres.

6.1. The case of positive Euler number. We will need the following result [14, Theorem 2.3].

Theorem 6.1. Let $\pi: S \rightarrow C$ be an elliptic surface. If the Euler number of $S$ is positive, then $\pi$ induces an isomorphism $\pi_{1}(S) \rightarrow \pi_{1}^{\text {orb }}(C)$.

Lemma 6.2. (a) Let $S$ be an elliptic surface with finite fundamental group. Then, the loop space homology of the universal covering of $S$ with rational coefficients grows exponentially.

(b) Let $S$ be a simply connected elliptic surface and let $f_{\infty}$ denote a regular fibre. Then, the loop space homology of $S-f_{\infty}$ with rational coefficients grows exponentially.

Proof. We will use the following fact proved by J.B. Friedlander and S. Halperin [13, Corollary 1.3]. Let $S$ be a 1-connected finite CW-complex. If the loop space homology of $S$ with rational coefficients grows subexponentially, then

$$
\sum_{k \geq 1} 2 k \operatorname{dim}\left(\pi_{2 k}(S) \otimes \mathbb{Q}\right) \leq n,
$$

where $n$ is the largest integer for which $H^{n}(S, \mathbb{Q}) \neq 0$.

To prove (a) observe that the Euler characteristic of $\widetilde{S}$ is given by $12 d$, where $d$ is a positive integer [14, Proposition 3.21]. Hence $b_{2}(\widetilde{S})=12 d-2>2$ and by the Hurewicz theorem $\operatorname{dim}\left(\pi_{2}(\widetilde{S}) \otimes \mathbb{Q}\right)>2$. Using the result mentioned above it follows that $S$ has the desired property.

Let us prove item (b). Observe first that $S-f_{\infty}$ is simply connected. Since $H_{1}(S)=H_{3}(S)=H_{1}\left(S-f_{\infty}\right)=0$, the Mayer-Vietoris sequence gives:

$$
0 \rightarrow \mathbb{Z}^{3} \rightarrow H_{2}\left(S-f_{\infty}\right) \oplus \mathbb{Z} \rightarrow H_{2}(S) \rightarrow \mathbb{Z}^{3} \rightarrow \mathbb{Z}^{2} \rightarrow 0
$$

It follows that $H_{2}\left(S-f_{\infty}\right)$ is a free abelian group of rank $b_{2}(S)+1$. One can deduce as above that $S-f_{\infty}$ has the desired property. 
We now prove:

Theorem 6.3. Let $\pi: S \rightarrow C$ be an elliptic surface. If the Euler number of $S$ is positive, then any $C^{\infty}$ Riemannian metric on $S$ has positive topological entropy.

Proof. We split the proof into several cases.

(1) $\pi_{1}^{\text {orb }}(C)$ is finite. By Theorem 6.1 $\pi_{1}(S)$ is finite. By Lemma 6.2 and Theorem $\mathrm{C}$ the topological entropy of any $C^{\infty}$ metric on $S$ is positive.

(2) $\pi_{1}^{\text {orb }}(C)$ is infinite, i.e., $\chi^{\text {orb }}(C) \leq 0$. If $\chi^{\text {orb }}(C)<0$, then $C$ is hyperbolic and $\pi_{1}^{\text {orb }}(C)$ contains a free subgroup of rank two. It follows from Theorem 6.1 that $\pi_{1}(S)$ grows exponentially and hence any metric on $S$ has positive topological entropy. If $\chi^{\mathrm{orb}}(C)=0$, then $C$ has a finite orbifold covering $C_{0}$ which has no multiple points and is a 2-torus. The finite cover $C_{0}$ induces a finite covering of the elliptic surface that we denote by $S_{0}$. By Theorem 2.16 in [14], $S_{0}$ is diffeomorphic to the fibre connected sum of $\mathbb{T}^{2} \times \mathbb{T}^{2}$ with a simply connected elliptic surface $S_{1}$ with no multiple fibres.

We can picture $\widetilde{S_{0}}$, the universal covering of $S_{0}$, as $\mathbb{T}^{2} \times \mathbb{R}^{2}$ fibre connected sum with infinitely many copies of $S_{1}$ as follows. Consider $\mathbb{T}^{2}$ (as is customary) given by the square with vertices $(0,0),(1,0),(1,1)$ and $(0,1)$ with the sides identified. At each fibre $\mathbb{T}^{2} \times\{(1 / 2+m, 1 / 2+n)\}$ consider the fibre connected sum with $S_{1}$ for all integers $m$ and $n$. The result is $\widetilde{S_{0}}$. Let $X$ be $S_{1}$ minus an open neighborhood of a regular fibre. Let us see that $X$ lives inside $\widetilde{S_{0}}$ as a retract. Consider a diffeomorphism of $\mathbb{R}^{2}$ that maps the points $(1 / 2+m, 1 / 2+$ $n)$ onto the points $(0, m)$. This induces a diffeomorphism of $\widetilde{S_{0}}$. Now retract $\mathbb{R}^{2}$ over the set $\left[-\frac{1}{2}, \frac{1}{2}\right] \times \mathbb{R}$ in the obvious way. This induces a retraction of $\widetilde{S_{0}}$. Now we can "fold" the strip onto the square $\left[-\frac{1}{2}, \frac{1}{2}\right] \times\left[-\frac{1}{2}, \frac{1}{2}\right]$ in such a way that the points $(0, m)$ all go to the origin preserving orientation. This describes a retraction of $\mathbb{R}^{2}$ over the square $\left[-\frac{1}{2}, \frac{1}{2}\right] \times\left[-\frac{1}{2}, \frac{1}{2}\right]$ which induces a retraction of $\widetilde{S}_{0}$ onto $X$.

By Lemma 6.2 and Corollary 4.4 the topological entropy of any $C^{\infty}$ metric on $S_{0}$ (and thus of any metric on $S$ ) is positive.

6.2. The case of zero Euler number. We will need the following result [14, Lemma 7.3, Proposition 7.4, Proposition 7.5].

Proposition 6.4. Let $\pi: S \rightarrow C$ be an elliptic fibration with Euler number zero. If the base orbifold is flat or hyperbolic (i.e. $\pi_{1}^{\mathrm{orb}}(C)$ is infinite), then there is an exact sequence

$$
0 \rightarrow \mathbb{Z} \oplus \mathbb{Z} \stackrel{i_{*}}{\rightarrow} \pi_{1}(S) \stackrel{\pi_{*}}{\rightarrow} \pi_{1}^{\text {orb }}(C) \rightarrow\{1\}
$$

where $i$ is the inclusion of a general fibre of $\pi$ into $S$. In case the base orbifold $C$ is spherical and good, then there is an exact sequence

$$
\mathbb{Z} \rightarrow \mathbb{Z} \oplus \mathbb{Z} \rightarrow \pi_{1}(S) \rightarrow \pi_{1}^{\text {orb }}(C) \rightarrow\{1\}
$$


In case the base orbifold $C$ is bad, then $S$ is either a ruled surface over an elliptic base $\left(\pi_{1}(S) \cong \mathbb{Z} \oplus \mathbb{Z}\right)$ or $S$ is a Hopf surface with $\pi_{1}(S) \cong \mathbb{Z} \oplus \mathbb{Z} / n \mathbb{Z}$ for some integer $n \geq 1$.

Let $\kappa$ be the Kodaira dimension.

Theorem 6.5. Let $\pi: S \rightarrow C$ be an elliptic fibration with Euler number zero. Suppose that $C$ is a good orbifold. The following are equivalent:

(1) $S$ admits a smooth Riemannian metric $g$ with $\mathrm{h}_{\mathrm{top}}(g)=0$;

(2) $S$ admits a geometric structure modelled on $S^{2} \times \mathbb{E}^{2}\left(\kappa=-\infty, b_{1}\right.$ even $), \mathbb{E}^{4}$ $\left(\kappa=0, b_{1}\right.$ even $), S^{3} \times \mathbb{E}^{1}\left(\kappa=-\infty, b_{1}\right.$ odd $)$ or $\mathrm{Nil}^{3} \times \mathbb{E}^{1}\left(\kappa=0, b_{1}\right.$ odd $)$.

Proof. The proof is based on the following result due to C.T.C Wall.

Theorem 6.6 (Theorem 7.4 in [40]). An elliptic surface $S$ without singular fibres has a geometric structure compatible with the complex structure of $S$ if and only if its base $C$ is a good orbifold. The type of the structure is determined as follows:

\begin{tabular}{cccc}
\hline$\kappa$ & $-\infty$ & 0 & 1 \\
\hline$b_{1}$ even & $S^{2} \times \mathbb{E}^{2}$ & $\mathbb{E}^{4}$ & $\mathbb{E}^{2} \times \mathbb{H}^{2}$ \\
$b_{1}$ odd & $S^{3} \times \mathbb{E}^{1}$ & $\mathrm{Nil}^{3} \times \mathbb{E}^{1}$ & $\widetilde{\mathrm{SL}}_{2} \times \mathbb{E}^{1}$ \\
\hline
\end{tabular}

Since we are assuming that $S$ has zero Euler number and that $C$ is a good orbifold, Wall's theorem says that $S$ admits a geometric structure as above. Lemma 5.2 ensures that if $S$ admits the geometries $\mathbb{E}^{2} \times \mathbb{H}^{2}$ and $\widetilde{S L}_{2} \times \mathbb{E}^{1}$, then $\pi_{1}(S)$ has exponential growth and the theorem readily follows from Proposition 5.1 .

We conclude this section by noting that elliptic surfaces with Kodaira dimension $-\infty$ and 0 can be completely classified [14, Proposition 3.23] and they are usually refer to as "not honestly" elliptic surfaces. Hence we can easily determine the surfaces appearing in Theorem 6.5.

(1) If $S$ admits a geometric structure modelled on $S^{2} \times \mathbb{E}^{2}\left(\kappa=-\infty, b_{1}\right.$ even), then $S$ is a ruled surface of genus 1 .

(2) If $S$ admits a geometric structure modelled on $\mathbb{E}^{4}\left(\kappa=0, b_{1}\right.$ even), then $S$ is torus or a hyperelliptic surface.

(3) If $S$ admits a geometric structure modelled on $S^{3} \times \mathbb{E}^{1}\left(\kappa=-\infty, b_{1}\right.$ odd $)$, then $S$ is a Hopf surface.

(4) If $S$ admits a geometric structure modelled on $\mathrm{Nil}^{3} \times \mathbb{E}^{1}\left(\kappa=0, b_{1}\right.$ odd $)$, then $S$ is a Kodaira surface, or a Kodaira surface modulo a finite group. 


\section{Surfaces of Kodaira dimension $-\infty$}

\subsection{Surfaces with a global spherical shell.}

Proposition 7.1. A compact surface with a global spherical shell admits no metric with zero topological entropy.

Proof. A compact surface $S$ with a global spherical shell is diffeomorphic to a connected sum of $S^{3} \times S^{1}$ with $b_{2}(S)$ copies of $\overline{\mathbb{C P}}^{2}\left(b_{2}(S)>0\right)$.

We can regard $\widetilde{S}$, the universal covering of $S$, as $S^{3} \times \mathbb{R}$ connected sum with infinitely many copies of $\overline{\mathbb{C} P^{2}}$ as follows. Consider $S^{1}$ as $[0,1]$ with the endpoints identified and fix a point $x \in S^{3}$. At each point $(x, 1 / 2+n) \in S^{3} \times \mathbb{R}$ consider the connected sum with $\overline{\mathbb{C} P^{2}}$ for all integers $n$. Let $X_{k}$ be the connected sum of $k$ copies of $\overline{\mathbb{C} P^{2}}$ with a small open ball around a point removed. For any $k, X_{k}$ lives inside $\widetilde{S}$ as a retract. If $k \geq 3$, the loop space homology of $X_{k}$ with rational coefficients grows exponentially and thus, by Corollary 4.4, the topological entropy of any $C^{\infty}$ metric on $S$ is positive.

\subsection{Hopf surfaces.}

Proposition 7.2. Any compact Hopf surface admits a smooth metric with zero topological entropy.

Proof. Let $H$ be a finite subgroup of $U(2)$ that acts freely on $S^{3} \subset \mathbb{C}^{2}$. According to M. Kato [23] any compact Hopf surface $S$ is diffeomorphic to one of the following:

(1) $S^{1} \times\left(S^{3} / H\right)$;

(2) $\left(S^{3} / H\right)$-bundle over $S^{1}$ whose transition function $S^{3} / H \rightarrow S^{3} / H$ is an involution. In fact, the bundles are diffeomorphic to $S^{1} \times\left(S^{3} / H\right)$ divided by an action of $\mathbb{Z}_{2}$ generated by

$$
([t],[q]) \mapsto([t+1 / 2],[u(q)])
$$

where $u$ is certain unitary matrix which normalizes $H$ and hence $[u(q)]$ is well defined.

Hence if we endow $S^{1} \times S^{3}$ with the canonical product metric we obtain a metric with zero entropy on $S^{1} \times\left(S^{3} / H\right)$ and since $\mathbb{Z}_{2}$ acts by isometries we also obtain a metric with zero entropy on any Hopf surface. In fact, $S$ admits a geometric structure modelled on $S^{3} \times \mathbb{E}$.

\subsection{Ruled surfaces.}

Proposition 7.3. A ruled surface $S$ over a Riemann surface $C$ of genus $g$ admits a smooth metric with zero topological entropy if and only if $g=0,1$.

Proof. It follows from the homotopy exact sequence of the fibration that if the fundamental group of $C$ grows exponentially, then the fundamental group of $S$ also grows 
exponentially. Hence if $S$ admits a smooth metric with zero topological entropy, then the genus of $C$ must be $\leq 1$.

From the differentiable viewpoint, there are only two $S^{2}$-bundles over $S^{2}$ : the trivial one $S^{2} \times S^{2}$ and the non-trivial one which is diffeomorphic to $\mathbb{C} P^{2} \# \overline{\mathbb{C} P^{2}}$. We explained in 35. how to construct smooth metrics with zero entropy on these manifolds.

Similarly, from the differentiable viewpoint, there are only two $S^{2}$-bundles over $\mathbb{T}^{2}$ [38]: a trivial bundle $E_{0}=\mathbb{T}^{2} \times S^{2}$ and a non-trivial bundle $E_{1}$. Clearly $E_{0}$ has a smooth metric with zero entropy.

The bundle $E_{1}$ can be described as follows (cf. [38, p. 310]). Let $\Gamma$ be the group isomorphic to $\mathbb{Z}_{2} \oplus \mathbb{Z}_{2}$ generated by the two diffeomorphisms of $S^{2} \times \mathbb{T}^{2}$ given by:

$$
\begin{aligned}
(p,[u]) & \mapsto\left(r_{z}(p),[u+1 / 2]\right), \\
(p,[u]) & \mapsto\left(r_{x}(p),[u+i / 2]\right),
\end{aligned}
$$

where $r_{z}$ is rotation by 180 degrees around the $z$-axis and $r_{x}$ is rotation by 180 degrees around the $x$-axis. Endow $S^{2} \times \mathbb{T}^{2}$ with the product metric. Since $\Gamma$ acts by isometries we see that $E_{1}$ admits a smooth metric with zero topological entropy. In fact $E_{1}$ admits a geometric structure modelled on $S^{2} \times \mathbb{E}^{2}$.

7.4. Inoue surfaces with $b_{2}=0$. They all have fundamental group with exponential growth and hence any metric has positive topological entropy. This can be checked directly from the explicit presentation of the fundamental groups given in [21. Alternatively, on account of Proposition 9.1 in [40 the Inoue surfaces admit geometric structures modelled on solvable groups and hence we can use Lemma 5.2.

\section{ENTROPy OF COMPLEX SURFACES}

We are now ready to complete the discussion of the minimal entropy problem for compact complex surfaces. The next proposition takes care of non-minimal surfaces.

Proposition 8.1. Let $S$ be a compact complex surface. If $S$ admits a $C^{\infty}$ metric with zero topological entropy, then $S$ must be minimal unless $S$ is diffeomorphic to $\mathbb{C} P^{2} \# \overline{\mathbb{C} P^{2}}$.

Proof. If $S$ is not minimal, it is diffeomorphic to $S^{\prime} \# \overline{\mathbb{C} P^{2}}$. Theorem D implies that the fundamental group of $S^{\prime}$ is either trivial or $\mathbb{Z}_{2}$. Moreover, the universal covering of $S^{\prime}$ must be homeomorphic to $S^{4}$ or $\mathbb{C} P^{2}$, otherwise the rational loop space homology of the universal covering of $S$ has exponential growth (cf. proof of part (a) Lemma 6.2 or [35]). Since $S$ is a complex surface, the result follows.

We can now directly combine Proposition 8.1 and Theorem B with the results of Sections 6 and 7 to obtain:

Theorem E. Let $S$ be a compact complex surface not of Kähler type. Modulo the gap in the classification of class VII surfaces we have: The minimal entropy of $S$ is zero and the following are equivalent: 
(1) The minimal entropy problem can be solved for $S$;

(2) $S$ admits a smooth metric $g$ with $\mathrm{h}_{\text {top }}(g)=0$;

(3) $S$ admits a geometric structure modelled on $S^{3} \times \mathbb{E}^{1}$ or $\mathrm{Nil}^{3} \times \mathbb{E}^{1}$;

(4) $S$ has $\kappa=-\infty, 0$ and is diffeomorphic to one of the following: a Hopf surface, a Kodaira surface, or a Kodaira surface modulo a finite group.

Similarly we can combine Proposition 8.1. Theorem A and the results of Sections 6 and 7 to obtain:

Theorem F. Let $S$ be a compact complex Kähler surface with Kodaira dimension $\kappa \leq 1$. We have: The minimal entropy of $S$ is zero and the following are equivalent:

(1) The minimal entropy problem can be solved for $S$;

(2) $S$ admits a smooth metric $g$ with $\mathrm{h}_{\mathrm{top}}(g)=0$;

(3) $S$ admits a geometric structure modelled on $\mathbb{C} P^{2}, S^{2} \times S^{2}, S^{2} \times \mathbb{E}^{2}$ or $\mathbb{E}^{4}$ or $S$ is diffeomorphic to $\mathbb{C} P^{2} \# \overline{\mathbb{C} P^{2}}$;

(4) $S$ has $\kappa=-\infty, 0$ and is diffeomorphic to one of the following: $\mathbb{C} P^{2}$, a ruled surface of genus 0 or 1 , a complex torus or a hyperelliptic surface.

Note that $\mathbb{C} P^{2} \# \overline{\mathbb{C} P^{2}}$ is the only non-minimal surface that admits a metric of zero entropy. It is also the only surface that admits a metric with zero entropy and no geometric structure.

\section{Appendix: Proof of Theorem 4.1 When $\partial X$ is NON-EMpty}

Proof. : Assume that the boundary of $X$ is non-empty. We have a collar of the boundary diffeomorphic to $\partial X \times[0,2)$. Let $Y$ be the manifold obtained by deleting $\partial X \times[0,1)$ from $X$. Of course, $Y$ is diffeomorphic to $X$. Now find a finite number of convex subsets of $X$ which cover $Y$. Call them $V_{\alpha}, 1 \leq \alpha \leq k_{0}$. Let $\mathbf{T}$ be a triangulation of $Y$. For a point $p \in Y$, let $F(p)$ be the closed cell of lowest dimension containing $p$ and let $O(p)$ be the union of all closed cells intersecting $F(p)$. Note that $O(p)$ is a compact subset of $Y$. Similarly, for any subset $K \subset Y$ one can define $F(Y)$ as the union of $F(p)$ for $p \in K$ and $O(K)$ as the union of $O(p)$ for $p \in K$. There is a positive number $\delta$ (depending on the covering $\left\{V_{\alpha}\right\}$ and $g$ ) such that, after taking some barycentric subdivisions, we can take $\mathbf{T}$ so that for every subset $K$ of diameter bounded by $\delta, O(K)$ is contained in one of the $V_{\alpha}$ 's.

Now for $x, y \in Y$ we define the open subset $\Omega_{k}$ of $\Omega\left(\cup V_{\alpha}, x, y\right)$ as the set of all paths $\omega$ in $\cup V_{\alpha}$ with $\omega(0)=x, \omega(1)=y$ so that for all $j$ between 1 and $2^{k}$,

$$
O\left(\left\{\omega\left(j-1 / 2^{k}\right), \omega\left(j / 2^{k}\right)\right\}\right) \cup \omega\left[j-1 / 2^{k}, j / 2^{k}\right]
$$

is contained in one of the $V_{\alpha}$ 's. It is easy to see that $\Omega(Y, x, y)$ is contained in the union of the $\Omega_{k}$ 's.

Let $B_{k}$ be the set of sequences $p_{0}, \ldots, p_{2^{k}}$ of points in $Y$ such that $p_{0}=x, p_{2^{k}}=y$ and for each $j$ between 1 and $2^{k} O\left(\left\{p_{j-1}, p_{j}\right\}\right)$ is contained in one of the $V_{\alpha}$ 's. Let $\Omega_{k}^{Y} \subset \Omega_{k}$ be the set of paths in $\Omega_{k}$ for which all intermediate $2^{k}-1$ points are in $Y$. Then $B_{k}$ is naturally identified with a subset of $\Omega_{k}^{Y}$ (an element of $B_{k}$ uniquely 
determines a broken geodesic which sends $j / 2^{k}$ to $p_{j}$ ) and it is actually a deformation retract of $\Omega_{k}^{Y}$.

Given a cycle representing a homology class in $\Omega(X, x, y)$ it can be represented by a cycle in $\Omega(Y, x, y)$ which is therefore contained in $\Omega_{k}^{Y}$ for some $k$. Therefore we can retract it to $B_{k}$. But $B_{k}$ is easily identified with a subset of $Y^{2^{k}-1}$. Moreover, under this identification if a point $\left(p_{1}, \ldots, p_{2^{k}-1}\right) \in B_{k}$, then the whole $F\left(p_{1}\right) \times \ldots \times F\left(p_{2^{k}-1}\right)$ is contained in $B_{k}$. This implies that $\mathbf{T}$ induces a cell decomposition in $B_{k}$. Hence the $i$-homology class can be represented by a combination of cells of dimension $i$. A cell in $B_{k}$ is a product of cells in each coordinate. The dimension of such a cell is the sum of the dimensions of the corresponding cells, of course. If the total dimension is $i$ then there can be at most $i$ cells of positive dimension. Since $X$ is simply connected there exists a smooth map $f: X \rightarrow X$ which is smoothly homotopic to the identity and which sends the union of the images of all the geodesic segments joining vertices in the triangulation to a point. We can moreover assume that $x$ and $y$ are fixed by $f$. The norm of the differential of $f$ is bounded since $X$ is compact and $f$ induces a map $\hat{f}: \Omega(X, x, y) \rightarrow \Omega(X, x, y)$ which is homotopic to the identity. Now paths belonging to an $i$-cell of $B_{k}$ are formed by pieces joining vertices of the triangulation and at most $2 i$ pieces in which one of the points is not a vertex. Under the map $\hat{f}$ the former are sent to a point and the latter to a path of length bounded in terms of the norm of the differential of $f$ and the diameter of the $V_{\alpha}$ 's. Therefore, there exists

a constant $C_{g}$ such that the image of the $i$-skeleton of $B_{k}$ is sent by $\hat{f}$ to the subset of paths with energy bounded by $C_{g} i$. The theorem follows.

\section{REFERENCES}

[1] J.W. Anderson, G.P. Paternain, The minimal entropy problem for 3-manifolds with zero simplicial volume, to appear in Asterisque.

[2] I. Babenko, Analytic properties of Poincaré series of a loop space, (Russian) Mat. Zametki 27 (1980) 751-765.

[3] I. Babenko, Topological entropy of geodesic flows on simply connected manifolds, and related problems, (Russian) Izv. Ross. Akad. Nauk. Ser. Mat. 61 (1997) 57-74.

[4] G. Besson, G. Courtois, S. Gallot, Entropies et rigidités des espaces localement symétriques de courbure strictement négative, Geom. Funct. Anal. 5 (1995) 731-799.

[5] F. A. Bogomolov, Classification of surfaces of class VII $I_{0}$ with $b_{2}=0$, Izv. Akad. Nauk SSSR Ser. Mat. 40 (1976) 273-288.

[6] F. A. Bogomolov, Surfaces of class VII 0 and affine geometry, Izv. Akad. SSSR Ser. Mat. 46 (1982) 710-761.

[7] L. Butler, Integrable geodesic flows on n-step nilmanifolds, J. Geom. Phys. 36 (2000) 315-323.

[8] L. Butler, Invariant metrics on nilmanifolds with positive topological entropy, to appear in Geom. Dedicata.

[9] J. Cheeger, M. Gromov, Collapsing Riemannian manifolds while keeping their curvature bounded I, J. Differential Geometry 23 (1986) 309-346.

[10] J. Cheeger, M. Gromov, Collapsing Riemannian manifolds while keeping their curvature bounded II, J. Differential Geometry 32 (1990) 269-298.

[11] E.I. Dinaburg, On the relations among various entropy characteristics of dynamical systems, Math. USSR Izv. 5 (1971) 337-378. 
[12] R.O. Filipkiewicz, Four dimensional geometries, Ph.D. thesis, University of Warwick, 1984.

[13] J.B. Friedlander, S. Halperin, An arithmetic characterization of the rational homotopy type of certain spaces, Invent. Math. 53 (1979) 117-133.

[14] R. Friedman, J. Morgan, Smooth four-manifolds and complex surfaces, Springer-Verlag, 1994.

[15] M. Gromov, Homotopical effects of dilatations, J. Diff. Geom. 13 (1978) 303-310.

[16] M. Gromov, Structures métriques pour les variétés Riemanniennes, by J. Lafontaine and P. Pansu, Editions Cedic/Fernand Nathan, Paris, 1981.

[17] M. Gromov, Metric structures for Riemannian and non-Riemannian spaces, Progress in Mathematics, 152, S.M. Bates (translator) 1999.

[18] M. Gromov, Entropy, homology and semialgebraic Geometry, Séminaire Bourbaki 38éme année, 1985-86 No 663, 225-240.

[19] M. Gromov, Volume and bounded cohomology, Publ. Math. I.H.E.S. 56 1-99.

[20] S. Halperin, M. Vigué-Poirrier, The homology of a free loop space, Pacific J. Math. 147 (1991) 311-324.

[21] M. Inoue, On surfaces of class VII $I_{0}$, Invent. Math. 24 (1974) 269-310.

[22] J. Jones, Cyclic homology and equivariant homology, Invent. Math. 87 (1987) 403-423.

[23] M. Kato, Topology of Hopf surfaces, J. Math. Soc. Japan 27 No. 2 (1975) 222-238.

[24] K. Kodaira, On compact complex analytic surfaces I, Ann. of Math. 71 (1960) 111-152. II, ibid, 77 (1963) 563-626, III, ibid, 78 (1963) 1-40.

[25] K. Kodaira, On the structure of compact complex analytic surfaces II, American Journal of Mathematics 88 (1966) 682-721.

[26] P. Lambrechts, The Betti numbers of the free loop space of a connected sum, J. London Math. Soc. (2) 64 (2001) 205-228.

[27] C. LeBrun, Kodaira Dimension and the Yamabe Problem, Comm. An. Geom. 7 (1999) 133-156.

[28] J. Li, S. T. Yau, F. Zheng, A simple proof of Bogomolov's theorem on class VII $I_{0}$-surfaces with $b_{2}=0$, Illinois J. Math. 34 (1990) 217-220.

[29] J. Li, S. T. Yau, F. Zheng, On projectively flat Hermitian manifolds, Comm. Anal. Geom. 2 (1994) 103-109.

[30] A. Manning, Topological entropy for geodesic flows, Ann. Math. 110 (1979), 567-573.

[31] J. Milnor, A note on curvature and fundamental group, Journal of Diff. Geometry 2 (1968) 1-7.

[32] I. Nakamura Towards classification of non-Kählerian complex surfaces, Sugaku Expositions 2, Number 2 (1989) 209-229.

[33] G.P. Paternain, Topological entropy for geodesic flows on fibre bundles over rationally hyperbolic manifolds, Proc. Amer. Math. Soc. 125 (1997) 2759-2765.

[34] G.P. Paternain, Geodesic flows, Progress in Mathematics, 180 Birkäuser 1999.

[35] G.P. Paternain, J. Petean, Minimal entropy and collapsing with curvature bounded from below, Invent. Math. 151 (2003) 415-450.

[36] D. Ruelle, An inequality for the entropy of differentiable maps, Bol. Soc. Brasil. Mat. 9 (1978) 83-87.

[37] T. Soma, The Gromov invariant of links, Invent. Math. 64 (1981) 445-454.

[38] T. Suwa, On ruled surfaces of genus 1, J. Math. Soc. Japan 21 (1969) 291-311.

[39] B. Totaro, Cheeger manifolds and the classification of biquotients, J. Differential Geom. 61 (2002) 397-451.

[40] C.T.C Wall, Geometric structures on compact complex analytic surfaces, Topology 25 (1986) $119-153$.

[41] J. Wolf, Growth of finitely generated solvable groups and curvature of Riemannian manifolds, J. Diff. Geom. 2 (1968) 421-446. 
Department of Pure Mathematics and Mathematical Statistics, University of Cambridge, Cambridge CB3 0WB, England

E-mail address: g.p.paternain@dpmms.cam.ac.uk

Cimat, A.P. 402, 36000, Guanajuato. Gto., México.

E-mail address: jimmy@cimat.mx 